\title{
WORK PLACE BULLYING AMONG NURSES IN PRIMARY HEALTH CARE CENTERS IN PORT SAID
}

\begin{abstract}
Mai EL-Ghareap Hassan ${ }^{1}$, Mona Abdel-Sabour Hassan ${ }^{2}$, Takwa Rashwan Mohamed Abd-El hady ${ }^{3}$

Lecturer of Family and Community Health Nursing, Faculty of Nursing, port said University, Egypt ${ }^{1}$

Professor of Family and Community Health Nursing, Faculty of Nursing, port said University, Egypt ${ }^{2}$

Lecturer of Nursing Administration, Faculty of Nursing, port said University, Egypt ${ }^{3}$

\section{ABSTRACT}

Background: Workplace bullying is common problem that threatens nursing profession, nurse's health and ability to work safely. Aim: to assess workplace bullying among nurses in primary health care centers in Port Said governorate. Setting: Study was conducted in seven primary health care centers representing districts of Port Said governorate. Sample: composed of 61 staff nurses working at health clinics in the randomly selected primary health care centers. Tools of data collection: Data were obtained through Negative Acts Questionnaire-Revised (NAQ-R). Results: Majority of staff nurses $(86.9 \%)$ had secondary nursing education and $82 \%$ were married. More than half of the studied nurses $(54.1 \%)$ were victims of workplace bullying, two third of nurses $(60.6 \%)$ had bullied by patients and patient relatives and more than one third (33.3\%) bullied by supervisor and manager. Conclusion: It was concluded from this study, more than half of nurses were victims of workplace bullying. There was no statistical significant relation between workplace bullying and socio-demographic data. Recommendation: Counseling sessions about consequences of workplace bullying should be held on regular base in health care centers.
\end{abstract}

Key Words: Workplace bullying, Nurses, primary health care centers 


\section{INTRODUCTION}

Workplace bullying (WPB) is universal problem spotted in different countries and several professions. It is form of aggression and actions that impeding a person's work (World Health Organization, 2012).There is no universally approved formal definition of workplace bullying, due to its complicated and multifaceted patterns (Branch, Ramsay \& Barker, 2013). Workplace bullying is a frequent hostile action by a person or group to another person (Sauer \& Mccoy, 2017).

The most generally approved definition of workplace bullying is meaning persecuting, and ignoring person that negatively influence his work tasks. Bullying victim label refers to repeated process to particular intimidate activity over a period (Ciby and Raya, 2015).This period is six months to differentiate between workplace bullying behavior and personal conflicts (Einarsen, Hoel, Zapf \& Cooper, 2011).

Workplace bullying in nursing has been a focus for researchers since the 1990, although workplace bullying was recognized over three decades ago. Nursing has recently identified workplace bullying as a serious, persistent, and devastating problem that needs open, continuing recognition and intervention (Johnston,Phanhtharath\& Jackson,2013) .The universal rate of workplace bullying among general workers ranges between $11 \%$ and $18 \%$ (Nielsen, Matthiesen \& Einarsen, 2010).Unfortunately $39.7 \%$ of nurses world widely were victims of workplace bullying (Spector, Zhou \& Che, 2013).

The negative behaviors of bullying are often classified into personal aspersion that includes use of frequent criticism, disrespect, humiliating orders to underestimate the target. Physical assaults or psychological threat, the misuse of power or strength are used to initiate a situation where the victim feels helpless to defend themselves or to have any actions (Tehrani, 2013).Work-related bullying behavior that involved hiding important information, removing responsibilities, unreasonable deadlines, undue workloads and insistent monitoring of work (Rai \& Agarwal, 2017).

Workplace bullying common forms among nurses are non-verbal aspersion, verbal abuse, hiding information, reducing activities, disrupting, conflicting, privacy failure, gloating, backstabbing and broken trust (Salin, 2015). The perpetrators in workplace exhibit a variety of harmful negative behaviors abusing his authority over the victim, resulting in extreme negative impact on the bully victim and whole health organization (Lee, Bernstein, Lee \& Nokes, 2014). 
On the bully victim it can lead to physical manifestations for the target as abdominal disorders, hypertension, frequent headaches and sleeping disorders, which adversely affect overall health (Ganz et. al,2015).Bullying affect whole organization extremely through increase in absenteeism rate, lower productivity, poor efficiency and job discontinuations (Karatza ,Zyga, Tziaferi \& Prezerakos ,2017).In addition to medication and treatment errors (Wright \& Khatri, 2015) low performance, sickness, and lack of enthusiasm (Hansen, Hogh, , Garde, \& Persson ,2014).

Primary health care is the cornerstone for building strong health care system that ensures positive health outcomes and health equity (Lawn, etal, 2008).Providing care services at primary health care centers necessitates close contact between nurses, patients and their families, often under challenging circumstances; however, workplace bullying hinder the provision of quality care services(Al-Turki, Afify\& AlAteeq, 2016).Several studies examined workplace bullying against nurses in settings other than primary health care center so, this study shed light on workplace bullying among nurses working in primary health care centers.

\section{Significance of the study}

Primary health care nurse encompass a broad range of practice with a very particular individual emphasis and broad responsibilities that include health promotion, early detection and intervention for well and at-risk communities throughout the lifespan. In primary health care centers nursing is more interacting with individuals, families and communities to achieve the common goal of health for all. According to (Howell, 2016) workplace bullying is more common among nurses than other health care professionals.

Nurse is the responsible for patient care, bullying toward them has negative consequence on patient's health, health care organizations and affects patients' safety (Ekici \& Beder, 2014). In Egypt a study was conducted in Cairo showed that nurses were mainly bullied by patients and relatives (Elewa \& El Banan, 2019). Additionally, many studies have reported that workplace bullying has alarming consequences on individual's health, team work and provided patient care (Duffy \& Sperry, 2012). Moreover (Jones,2017) reported that workplace bullying affect nursing profession and causing elevation of attrition rates, decreased productivity, nursing shortage and 
lowered job safety so, this research was conducted to assess workplace bullying among nurses in primary health care centers.

\section{AIM OF THE STUDY}

To assess workplace bullying among nurses in primary health care centers in Port Said.

\section{Research questions:}

- How prevalent was workplace bullying against nurses during the past six months?

- What are the perpetrators characteristics?

- Is there a relation between workplace bullying and socio-demographic characteristics of nurses?

\section{SUBJECTS AND METHOD}

\section{Study Design:}

Descriptive design was applied in this study to accomplish the aim of study.

\section{Study setting:}

The study was conducted in seven primary health care centers in Port Said governorate, from each district in Port Said governorate one primary health care center has been selected randomly. These health centers are Al-Kuwait health center which representing Elmanakh district, Omar Ben Elkatab health center which representing Elzohour district ,Fatma Elzahara health center which representing Eldawahy district,Seha-OUla health center which representing Elsharq district, Elarab health care center which representing Elarab district ,Port fouad awal health center which representing Port fouad district and Elraswa health care center which representing Elganoub district. The study was conducted at all health clinics that provide health care services by nurses in previously mentioned settings.

\section{Subjects:}

All nurses working in primary health care centers that mentioned before and work in the following clinics family planning, antenatal, dental, vaccination, comprehensive examination clinic, pediatrics, family medicine, emergency and adult clinics. Inclusion criteria:

- Staff nurses working in health care centers for more than six months.

\section{TOOL OF DATA COLLECTION}

Data collected using one tool. It was adopted from (Alaslawi, 2017). It is composed of three parts as follow: 


\section{Part (I):}

The first part was socio- demographic characteristics questionnaire. It was used to assess demographic characteristics of studied sample. It mainly composed of questions that include age, sex, religion, educational level, marital status, work site, years of experience in nursing profession, and years of experience in current health care centers.

\section{Part (II):}

The second part was The Negative Acts Questionnaire-Revised (NAQ-R). It was utilized to measure nurse's exposure to workplace bullying within the past six months. It was developed by (Einarsen, Hoel \& Notelaers, 2009) the Arabic translated version by (Alaslawi, 2017) was used in this study. It had two sections: the behavioral experience section and the self-labeling section.

NAQ-R Behavioral Experience Section: consists of 22 items categorized into three underlying dimensions. The first one named work-related bullying, it is composed of (7items) ; the second dimension is person-related bullying, it is composed of (12 items), and the third is physically intimidating bullying, it is composed (3items).

NAQ-R Self-Labeling Section: this section was one item. It was used to identify bullying victimization. Participants were asked if they had been bullied in the workplace, according to the global definition of workplace bullying. This item was aimed to detect any mismatch between the self-report of perceiving negative experiences and the self-labeling as bully victims based on the provided definition of bullying within previous six months.

\section{Scoring System:}

The Negative Acts Questionnaire-Revised (NAQ-R) items were scored 5, 4, 3, 2, and 1 for the respond "daily", "weekly", "monthly", "now and then", and "never" respectively. Total score of 33 or lower indicates that a participant is not being bullied at work, however the total score falls between 33 and 45 indicates occasional bullying, and the score over 45 is an indication of daily bullying.

\section{Part (III):}

The third part was victimization and perpetrators section. It was used to identify more information for better profile about victims and perpetrators. Only the respondents who labeled themselves as victims by answering (yes) to self-labeling section had access to the rest of these items. It is composed of 9 items the first six items about perpetrator's demographics characteristics as who is perpetrator, sex, number, 
education, age and the number of employees the bully supervises. The other three items about victimization profile as result of perpetrators actions are absenteeism with illness due to being bullied, intention to quit or transfer from their job and lower work performance.

\section{Validity:}

The tool validity is confirmed by jury panel of five academic experts in community health nursing field.

\section{Reliability}

Tool reliability was tested using Cronbach alpha coefficients test. Negative Acts Questionnaire-Revised scale was reliable and the internal consistency coefficient was (.90).

\section{Pilot Study:}

The pilot study was carried out on $10 \%$ of nurses which represent ( 7 nurses). The main goal of pilot study is to assess the accuracy, applicability, clarity, and to determine required time to fill in study tool. The study tool was filled in by nurses. The time needed for completion of the questionnaire ranged from 15 to 20 minutes. Pilot study sample wasn't included in the main study sample.

\section{Fieldwork:}

In each primary health care center the researcher met and explained the aim of study to nurses in a simple way, the consent was obtained before their participation in the current study. The researcher attended to primary health care center from 8:30 AM to 2 PM, each primary health care center was visited for two days per week. Data for the current study were collected through period started from 6 January 2019 to end of March 2019. Self-administered written questionnaire were filled out by nurses. Time needed for filling questionnaire ranged from 15 to 20 minutes.

\section{Administrative design}

Before conducting the study, a written letter explaining the aim of the study was directed from faculty of nursing port-said university to medical and nursing directors of primary health care centers, to ensure their cooperation and permission after explaining the aim of the study. 


\section{Ethical Consideration:}

Permission was taken from primary health care centers medical and nursing directors to carry out the study. The researcher explains the aim of the study to each nurse before participation. Consent was obtained from nurses, nurses assured that information will be kept confidential and they have the right to withdraw from the study without any consequences.

\section{Statistical analysis}

Data entry and statistical analysis were done using SPSS 20.0 statistical software package. Data were presented using descriptive statistics in the form of frequencies and percentages for qualitative variables, and means and standard deviations for quantitative variables. Qualitative categorical variables were compared using chi-square test. Statistical significance was considered at $p$-value $<0.05$.

\section{RESULT:}

Table (1): shows that all of studied nurses (100\%) were females, $34.4 \%$ of them their age ranged from 35 to $<40$ years old. Concerning marital status $82 \%$ of nurses were married, $83.6 \%$ were Muslims and $86.9 \%$ of them had secondary nursing education. As regard years of experience $42.6 \%$ of nurses had $\geq 20$ years of experience in nursing field and $49.2 \%$ of nurses had $\geq 10$ years of experience in current health center.

Table (2): reveals bullying behavior toward studied nurses. As regard work related bullying $36.1 \%$ of studied nurses were being exposed to an unmanageable workload now and then, moreover $26.2 \%$ of nurse's opinions and views were ignored; also $26.2 \%$ were pressured not to claim their right as sick leave. Regarding person related bullying $27.9 \%$ of nurses humiliated or ridiculed in connection with their work, $23.0 \%$ had exposed to repeated reminders of their errors or mistakes. According to physically intimidating bullying $37.7 \%$ of the studied nurses mentioned that being shouted or being the target of spontaneous anger. Data also indicated that, $29.5 \%$ of the studied nurses exposed to fingerpointing, invasion of personal space, and barring the way.

Table (3) shows workplace bullying victims, more than half of studied nurses $(54.1 \%)$ were victims of work place bullying, and $60.6 \%$ of nurses who had been bullied stated that they had been bullied by patients and patient relatives. As regard sex of perpetrator, $54.5 \%$ of nurses were bullied by both male $\&$ female perpetrator and $42.4 \%$ of nurses bullied by more than two perpetrators also, $21.2 \%$ of perpetrators were more educated than nurses.

Table (4) shows that $15.2 \%$ of the studied nurses had 1 to 6 days off work due to sickness as results of bullying, also $33.3 \%$ of nurses sometimes consider quitting or transferring from present job and $12.1 \%$ of nurses had lower productivity sometimes due to bullying. 
Table (5) reveals that no statistical significant relation between workplace bullying and studied nurses socio-demographic data.

Table (1): Socio-demographic characteristics of the studied nurses $(n=61)$

\begin{tabular}{|c|c|c|}
\hline Items & $\begin{array}{l}\text { No. } \\
\end{array}$ & $\%$ \\
\hline $\begin{array}{l}\text { Primary health care center } \\
\text { Elarab health care center } \\
\text { Kuwait health center } \\
\text { Omar Bin Al - Khattab health center } \\
\text { Seha-OUla health center } \\
\text { Fatma Elzahara health center } \\
\text { Port fouad awal health center } \\
\text { Elraswa health care center }\end{array}$ & $\begin{array}{l}9 \\
12 \\
10 \\
6 \\
9 \\
9 \\
6 \\
\end{array}$ & $\begin{array}{l}14.8 \\
19.7 \\
16.4 \\
9.8 \\
14.8 \\
14.8 \\
9.8 \\
\end{array}$ \\
\hline $\begin{array}{l}\text { Sex } \\
\text { Female } \\
\text { Male }\end{array}$ & $\begin{array}{l}61 \\
0\end{array}$ & $\begin{array}{l}100.0 \\
0.0\end{array}$ \\
\hline $\begin{array}{l}\text { Age } \\
20-<25 \\
25-<30 \\
35-<40 \\
40+\end{array}$ & $\begin{array}{l}7 \\
12 \\
21 \\
21\end{array}$ & $\begin{array}{l}11.5 \\
19.7 \\
34.4 \\
34.4\end{array}$ \\
\hline $\begin{array}{l}\text { Religion } \\
\text { Muslim } \\
\text { Christian } \\
\end{array}$ & $\begin{array}{l}51 \\
10 \\
\end{array}$ & $\begin{array}{l}83.6 \\
16.4 \\
\end{array}$ \\
\hline $\begin{array}{l}\text { Social status } \\
\text { Single } \\
\text { Married } \\
\text { Divorced } \\
\end{array}$ & $\begin{array}{l}9 \\
50 \\
2 \\
\end{array}$ & $\begin{array}{l}14.8 \\
82.0 \\
3.3\end{array}$ \\
\hline $\begin{array}{l}\text { Level of education } \\
\text { Secondary Nursing } \\
\text { Technical technician } \\
\text { Bachelor of Nursing }\end{array}$ & $\begin{array}{l}53 \\
6 \\
2 \\
\end{array}$ & $\begin{array}{l}86.9 \\
9.8 \\
3.3 \\
\end{array}$ \\
\hline $\begin{array}{l}\text { Work site } \\
\text { Family Planning clinic } \\
\text { Adult clinic } \\
\text { Vaccinations clinic } \\
\text { Pediatrics clinic } \\
\text { Dental clinic } \\
\text { Family medicine } \\
\text { Antenatal clinic } \\
\text { emergency clinic } \\
\text { comprehensive examination clinic }\end{array}$ & $\begin{array}{l}11 \\
8 \\
20 \\
1 \\
5 \\
4 \\
6 \\
5 \\
1 \\
\end{array}$ & $\begin{array}{l}18.0 \\
13.1 \\
32.8 \\
1.6 \\
8.2 \\
6.6 \\
9.8 \\
8.2 \\
1.6 \\
\end{array}$ \\
\hline $\begin{array}{l}\text { The sex of the patients you work with most often } \\
\text { Male } \\
\text { Female } \\
\text { Male \& Female }\end{array}$ & $\begin{array}{l}0 \\
13 \\
48\end{array}$ & $\begin{array}{l}0.0 \\
21.3 \\
78.7\end{array}$ \\
\hline $\begin{array}{l}\text { Years of experience in nursing profession } \\
<10 \\
10-<20 \\
\geq 20\end{array}$ & $\begin{array}{l}12 \\
23 \\
26 \\
\end{array}$ & $\begin{array}{l}19.7 \\
37.7 \\
42.6 \\
\end{array}$ \\
\hline $\begin{array}{l}\text { Years of experience in current center } \\
<5 \\
5-<10 \\
\geq 10 \\
\end{array}$ & $\begin{array}{l}21 \\
10 \\
30 \\
\end{array}$ & $\begin{array}{l}34.4 \\
16.4 \\
49.2 \\
\end{array}$ \\
\hline
\end{tabular}


Table (2): Bullying behaviors against studied nurses in primary health care center $(n=61)$

\begin{tabular}{|c|c|c|c|c|c|c|c|c|c|c|}
\hline \multirow[t]{2}{*}{ NAQ-R } & \multicolumn{2}{|c|}{ Never } & \multicolumn{2}{|c|}{$\begin{array}{c}\text { Now \& } \\
\text { then }\end{array}$} & \multicolumn{2}{|c|}{ Monthly } & \multicolumn{2}{|c|}{ Weekly } & \multicolumn{2}{|c|}{ Daily } \\
\hline & No. & $\%$ & No. & $\%$ & No. & $\%$ & No. & $\%$ & No. & $\%$ \\
\hline $\begin{array}{l}\text { Work related bullying } \\
\text { Someone withholding information which affects your } \\
\text { performance }\end{array}$ & 45 & 73.8 & 12 & 19.7 & 1 & 1.6 & 1 & 1.6 & 2 & 3.3 \\
\hline $\begin{array}{l}\text { Being ordered to do work below your level of } \\
\text { competence. }\end{array}$ & 46 & 75.4 & 12 & 19.7 & 1 & 1.6 & 0 & 0.0 & 2 & 3.3 \\
\hline Having your opinions and views ignored. & 45 & 73.8 & 16 & 26.2 & 0 & 0.0 & 0 & 0.0 & 0 & 0.0 \\
\hline $\begin{array}{l}\text { Being given tasks with unreasonable or impossible } \\
\text { targets or deadlines }\end{array}$ & 49 & 80.3 & 11 & 18.0 & 0 & 0.0 & 0 & 0.0 & 1 & 1.6 \\
\hline Excessive monitoring of your work. & 42 & 68.9 & 16 & 26.2 & 2 & 3.3 & 0 & 0.0 & 1 & 1.6 \\
\hline $\begin{array}{l}\text { Pressure not to claim something which by right you } \\
\text { are entitled to (e.g., sick leave, holiday entitlement, } \\
\text { travel expenses). }\end{array}$ & 44 & 72.1 & 16 & 26.2 & 0 & 0.0 & 0 & 0.0 & 1 & 1.6 \\
\hline Being exposed to an unmanageable workload. & 37 & 60.7 & 22 & 36.1 & 0 & 0.0 & 1 & 1.6 & 1 & 1.6 \\
\hline $\begin{array}{l}\text { Person related bullying } \\
\text { Being humiliated or ridiculed in connection with } \\
\text { your work }\end{array}$ & 41 & 67.2 & 17 & 27.9 & 0 & 0.0 & 1 & 1.6 & 2 & 3.3 \\
\hline $\begin{array}{l}\text { Having key areas of responsibility removed or } \\
\text { replaced with more trivial or unpleasant tasks. }\end{array}$ & 47 & 77.0 & 14 & 23.0 & 0 & 0.0 & 0 & 0.0 & 0 & 0.0 \\
\hline Spreading of gossip and rumors about you. & 46 & 75.4 & 9 & 14.8 & 6 & 9.8 & 0 & 0.0 & 0 & 0.0 \\
\hline Being ignored or excluded & 54 & 88.5 & 7 & 11.5 & 0 & 0.0 & 0 & 0.0 & 0 & 0.0 \\
\hline $\begin{array}{l}\text { Having insulting or offensive remarks made about } \\
\text { your person (i.e., habits and background), your } \\
\text { attitudes or your private life }\end{array}$ & 51 & 83.6 & 8 & 13.1 & 2 & 3.3 & 0 & 0.0 & 0 & 0.0 \\
\hline $\begin{array}{l}\text { Hints or signals from others that you should quit your } \\
\text { Job }\end{array}$ & 42 & 68.9 & 19 & 31.1 & 0 & 0.0 & 0 & 0.0 & 0 & 0.0 \\
\hline Repeated reminders of your errors or mistakes. & 47 & 77.0 & 14 & 23.0 & 0 & 0.0 & 0 & 0.0 & 0 & 0.0 \\
\hline $\begin{array}{l}\text { Being ignored or facing a hostile reaction when you } \\
\text { approach. }\end{array}$ & 56 & 91.8 & 5 & 8.2 & 0 & 0.0 & 0 & 0.0 & 0 & 0.0 \\
\hline Persistent criticism of your work and effort. & 50 & 82.0 & 11 & 18.0 & 0 & 0.0 & 0 & 0.0 & 0 & 0.0 \\
\hline $\begin{array}{l}\text { Practical jokes carried out by people you don't get on } \\
\text { with }\end{array}$ & 48 & 78.7 & 13 & 21.3 & 0 & 0.0 & 0 & 0.0 & 0 & 0.0 \\
\hline Having allegations made against you. & 54 & 88.5 & 7 & 11.5 & 0 & 0.0 & 0 & 0.0 & 0 & 0.0 \\
\hline Being the subject of excessive teasing and sarcasm. & 52 & 85.2 & 5 & 8.2 & 4 & 6.6 & 0 & 0.0 & 0 & 0.0 \\
\hline $\begin{array}{l}\text { Physically intimidating bullying } \\
\text { Being shouted at or being the target of spontaneous } \\
\text { anger. }\end{array}$ & 35 & 57.4 & 23 & 37.7 & 0 & 0.0 & 1 & 1.6 & 2 & 3.3 \\
\hline $\begin{array}{l}\text { Intimidating behavior such as finger } \\
\text { pointing,invasion of personal space, shoving, } \\
\text { blocking/barring } \\
\text { the way }\end{array}$ & 42 & 68.9 & 18 & 29.5 & 0 & 0.0 & 1 & 1.6 & 0 & 0.0 \\
\hline Threats of violence or physical abuse or actual abuse. & 45 & 73.8 & 13 & 21.3 & 0 & 0.0 & 2 & 3.3 & 1 & 1.6 \\
\hline
\end{tabular}


Table (3): $\quad$ Perpetrators characteristics as reported by bullied nurses

\begin{tabular}{|c|c|c|}
\hline & No. & $\%$ \\
\hline $\begin{array}{l}\text { Nurses victims at work based on global definition of work place } \\
\text { bullying } \\
\text { No } \\
\text { Yes }\end{array}$ & $\begin{array}{l}28 \\
33\end{array}$ & $\begin{array}{l}45.9 \\
\mathbf{5 4 . 1}\end{array}$ \\
\hline $\begin{array}{l}\text { If your answer Yes: }(\mathbf{n}=\mathbf{3 3}) \\
\text { Who you were bullied by }(\mathbf{n}=\mathbf{3 3}) \\
\text { superiors/managers in the organization } \\
\text { Colleagues } \\
\text { Clients/ patients and patient relatives }\end{array}$ & $\begin{array}{l}11 \\
2 \\
20\end{array}$ & $\begin{array}{l}33.3 \\
6.1 \\
60.6\end{array}$ \\
\hline $\begin{array}{l}\text { Please state the sex of your perpetrator/s: }(\mathbf{n}=\mathbf{3 3}) \\
\text { Male perpetrator } \\
\text { Female perpetrator } \\
\text { Male \& Female perpetrator }\end{array}$ & $\begin{array}{l}7 \\
8 \\
18\end{array}$ & $\begin{array}{l}21.2 \\
24.2 \\
54.5\end{array}$ \\
\hline $\begin{array}{l}\text { Please state the number of your perpetrator/s: }(n=33) \\
1 \\
2 \\
>2\end{array}$ & $\begin{array}{l}9 \\
10 \\
14\end{array}$ & $\begin{array}{l}27.3 \\
30.3 \\
42.4\end{array}$ \\
\hline $\begin{array}{l}\text { Does your main perpetrator have the same or more or less } \\
\text { education than you?: }(\mathbf{n}=\mathbf{3 3}) \\
\text { Same } \\
\text { Less } \\
\text { More } \\
\text { don`t know }\end{array}$ & $\begin{array}{l}1 \\
1 \\
7 \\
24\end{array}$ & $\begin{array}{l}3.0 \\
3.0 \\
21.2 \\
72.7\end{array}$ \\
\hline $\begin{array}{l}\text { Please state approximately the age of your main perpetrator/s: } \\
\text { Male perpetrator: }(\mathbf{n}=\mathbf{3 3}) \\
18:<23 \\
23:<28 \\
28:<32 \\
32:<37 \\
37:<42 \\
42:<47 \\
47:<52 \\
>52\end{array}$ & $\begin{array}{l}1 \\
1 \\
6 \\
7 \\
14 \\
2 \\
1 \\
1 \\
\end{array}$ & $\begin{array}{l}3.0 \\
3.0 \\
18.2 \\
21.2 \\
42.4 \\
6.1 \\
3.0 \\
3.0\end{array}$ \\
\hline $\begin{array}{l}\text { Approximately, how many employees the main bully supervises } \\
1: 9 \\
>100 \\
\text { don't know }\end{array}$ & $\begin{array}{l}2 \\
3 \\
28\end{array}$ & $\begin{array}{l}6.1 \\
9.1 \\
84.8\end{array}$ \\
\hline
\end{tabular}


Table (4): Distribution of the bullied nurses according to victimization work profile

\begin{tabular}{|l|l|l||}
\hline Items & No. & $\%$ \\
\hline In the last 6 months, how many days approximately have & & \\
you been off work with illness due to being bullied? & 5 & 15.2 \\
$1: 6$ & 1 & 3.0 \\
$7: 13$ & 3 & 9.1 \\
$14: 21$ & 24 & 72.7 \\
No days off & & \\
\hline In the last 6 months, have you considered quitting or & \\
transferring from your present job due to being bullied? & 17 & 51.5 \\
Never & 3 & 9.1 \\
Rarely & 11 & 33.3 \\
Sometimes & 2 & 6.1 \\
Often & & \\
\hline In the last 6 months, has your productivity been lower & \\
than expected due to being bullied? & 24 & 72.8 \\
Never & 5 & 15.1 \\
Rarely & 4 & 12.1 \\
Sometimes & \\
\hline
\end{tabular}

Table (5): Relationship between work place bullying and socio-demographic data $(\mathrm{n}=61)$

\begin{tabular}{|c|c|c|c|c|c|c|}
\hline \multirow{3}{*}{ Items } & \multicolumn{4}{|c|}{ Work place bulling } & \multirow{3}{*}{$\chi^{2}$} & \multirow{3}{*}{$\mathbf{p}$} \\
\hline & \multicolumn{2}{|c|}{$\begin{array}{l}\text { No } \\
(\mathrm{n}=28)\end{array}$} & \multicolumn{2}{|c|}{$\begin{array}{l}\text { Yes } \\
(n=33)\end{array}$} & & \\
\hline & No. & $\%$ & No. & $\%$ & & \\
\hline \multirow{8}{*}{$\begin{array}{l}\text { Primary health care center } \\
\text { Elarab health care center } \\
\text { Kuwait health care center } \\
\text { Omar Bin Al - Khattab health care center } \\
\text { Seha-OUla health care center } \\
\text { Fatma Elzahara health care center } \\
\text { Port fouad awal health care center } \\
\text { Elraswa health care center }\end{array}$} & & & & & \multirow{8}{*}{2.987} & \multirow{8}{*}{0.855} \\
\hline & 6 & 21.4 & 3 & 9.1 & & \\
\hline & 5 & 17.9 & 7 & 21.2 & & \\
\hline & 4 & 14.3 & 6 & 18.2 & & \\
\hline & 2 & 7.1 & 4 & 12.1 & & \\
\hline & 4 & 14.3 & 5 & 15.2 & & \\
\hline & 5 & 17.9 & 4 & 12.1 & & \\
\hline & 2 & 7.1 & 4 & 12.1 & & \\
\hline \multirow{3}{*}{$\begin{array}{l}\text { Sex } \\
\text { Male } \\
\text { Female }\end{array}$} & & & & & \multirow[b]{3}{*}{-} & \multirow[b]{3}{*}{-} \\
\hline & 0 & 0.0 & 0 & 0.0 & & \\
\hline & 28 & $\begin{array}{l}100 . \\
0\end{array}$ & 33 & $\begin{array}{l}100 . \\
0\end{array}$ & & \\
\hline \multirow{5}{*}{$\begin{array}{l}\text { Age } \\
20-<25 \\
25-<30 \\
35-<40 \\
40+\end{array}$} & & & & & \multirow{5}{*}{1.597} & \multirow{5}{*}{0.683} \\
\hline & 4 & 14.3 & 3 & 9.1 & & \\
\hline & 4 & 14.3 & 8 & 24.2 & & \\
\hline & 9 & 32.1 & 12 & 36.4 & & \\
\hline & 11 & 39.3 & 10 & 30.3 & & \\
\hline \multirow{2}{*}{$\begin{array}{l}\text { Religion } \\
\text { Muslim }\end{array}$} & & & & & \multirow{3}{*}{0.081} & \multirow{3}{*}{1.000} \\
\hline & 23 & 82.1 & 28 & 84.8 & & \\
\hline Christian & 5 & 17.9 & 5 & 15.2 & & \\
\hline \multirow{4}{*}{$\begin{array}{l}\text { Social status } \\
\text { Single } \\
\text { Married } \\
\text { Divorced }\end{array}$} & & & & & \multirow{4}{*}{1.473} & \multirow{4}{*}{0.640} \\
\hline & 4 & 14.3 & 5 & 15.2 & & \\
\hline & 24 & 85.7 & 26 & 78.8 & & \\
\hline & 0 & 0.0 & 2 & 6.1 & & \\
\hline
\end{tabular}




\begin{tabular}{|c|c|c|c|c|c|c|}
\hline $\begin{array}{l}\text { Level of education } \\
\text { Secondary Nursing } \\
\text { Technical technician } \\
\text { Bachelor of Nursing }\end{array}$ & $\begin{array}{l}25 \\
2 \\
1\end{array}$ & $\begin{array}{l}89.3 \\
7.1 \\
3.6\end{array}$ & $\begin{array}{l}28 \\
4 \\
1\end{array}$ & $\begin{array}{l}84.8 \\
12.1 \\
3.0\end{array}$ & 0.678 & $\mid 0.840$ \\
\hline $\begin{array}{l}\text { Work site } \\
\text { Family Planning clinic } \\
\text { Adult clinic } \\
\text { Vaccinations clinic } \\
\text { Pediatrics clinic } \\
\text { Dental clinic } \\
\text { Family medicine } \\
\text { Antenatal clinic } \\
\text { Emergency clinic } \\
\text { comprehensive examination clinic }\end{array}$ & $\begin{array}{l}5 \\
5 \\
5 \\
1 \\
1 \\
4 \\
4 \\
3 \\
0\end{array}$ & $\begin{array}{ll}17.9 \\
17.9 \\
17.9 \\
3.6 \\
3.6 \\
14.3 \\
14.3 \\
10.7 \\
0.0\end{array}$ & $\begin{array}{l}6 \\
3 \\
15 \\
0 \\
4 \\
0 \\
2 \\
2 \\
1\end{array}$ & $\begin{array}{l}18.2 \\
9.1 \\
45.5 \\
0.0 \\
12.1 \\
0.0 \\
6.1 \\
6.1 \\
3.0\end{array}$ & 13.412 & 0.066 \\
\hline $\begin{array}{l}\text { The sex of the patients you work with } \\
\text { most often } \\
\text { Male } \\
\text { Female } \\
\text { Male \& Female }\end{array}$ & \begin{tabular}{|l|}
0 \\
7 \\
21
\end{tabular} & $\begin{array}{l}0.0 \\
25.0 \\
75.0\end{array}$ & $\begin{array}{l}0 \\
6 \\
27\end{array}$ & $\begin{array}{l}0.0 \\
18.2 \\
81.8\end{array}$ & 0.420 & 0.517 \\
\hline $\begin{array}{l}\text { Years of experience in nursing } \\
\text { profession } \\
<10 \\
10-<20 \\
\geq 20\end{array}$ & \begin{tabular}{|l}
6 \\
10 \\
12
\end{tabular} & $\begin{array}{l}21.4 \\
35.7 \\
42.9\end{array}$ & $\begin{array}{l}6 \\
13 \\
14\end{array}$ & $\begin{array}{l}18.2 \\
39.4 \\
42.4\end{array}$ & 0.136 & 0.934 \\
\hline $\begin{array}{l}\text { Years of experience in the current } \\
\text { center } \\
<5 \\
5-<10 \\
\geq 10\end{array}$ & \begin{tabular}{|l}
12 \\
6 \\
10
\end{tabular} & $\begin{array}{l}42.9 \\
21.4 \\
35.7\end{array}$ & $\begin{array}{l}9 \\
4 \\
20\end{array}$ & $\begin{array}{l}27.3 \\
12.1 \\
60.6\end{array}$ & 3.777 & 0.151 \\
\hline
\end{tabular}

$\chi^{2}$ : Chi square test $\quad$ MC: Monte Carlo $\quad$ FE: Fisher Exact

$\mathrm{p}$ : $\mathrm{p}$ value for association between different categories.

\section{DISCUSSION}

Nurses are considered predominant and the corner stone in providing healthcare to individuals and community, unfortunately workplace bullying exists in almost all workplaces settings. Workplace bullying not only affect nurse, but also impacted the entire workplace environment, patient health and the whole organization. There are three types of negative workplace bullying which faced by the nurses, work related bullying; person related bullying and physically intimidating bullying.

As regard work related bullying as first source of negative act toward nurses table 2 showed that more than one third of nurses were exposed to unmanageable workload now \& then. This result goes in line with McMahon, MacCurtain, O'Sullivan, Murphy \& Turner 
(2013)study in Ireland who indicated that fifth of nurses had experienced unmanageable workload. Also, Butler, Prentiss, \& Benamor (2018) who studied prevalence of workplace bullying among registered nurse and clinical partner had reported that both exposed to unmanageable workload. On the other extreme study was conducted on 1152 nurses recruited in Tokyo by Yokoyama, Suzuki, Takai, Igarashi, Noguchi-Watanabe \& Yamamoto-Mitani(2016), stated that few nurses has been exposed to unmanageable workload. This may be due to increase work pressure in health care center due to varied patient needs, manpower shortage as primary health care centers in Port Said work with half of nursing capacity due to preparation for the comprehensive insurance system, frequent change in nurse's roles and lack of resources.

The current study results declared that more than one quarter of nurses were pressured not to claim their right as sick leave, holiday permission and travel costs. This result goes in line with El-Houfey, Abo El-Maged, Elserogy \& El Ansari (2015) who conducted a study in Egypt and revealed that more than one third of the nurses were forced not to use their rights as sick leave and holiday. This may be due to shortage of staff nurses and manager always invoke the need of staff nurses to fill the shortage.

Regarding person related bullying the present study results showed that almost one quarter of nurses had exposed to repeated reminders of their errors and mistakes as shown in table (2). This is consistent with Al-Ghabeesh \& Qattom (2019) study which conducted in Amman, Jordan and their results indicated that nurses always reminded with their errors and faults. In addition to Roy\& Khan (2020) who conducted descriptive study at Chattogram in Bangladesh among 183 nurses in eight health care facilities revealed that most of nurses exposed to repeated reminders of their errors. This study finding may be due to poor communication between nurse and management, unfair work assignment to fill shortage, increased workload and overburdening responsibilities.

The current results furthermore declared that more than one quarter of studied nurses being humiliated or ridiculed in connection with their work. This result consistent with Roy\& Khan (2020) who stated that majority of nurses were being humiliated in connection with their work, also they faced by ignoring opinions and views. This study finding may be due to majority of studied nurses had secondary nursing education, lack of appreciation of nurses' efforts and misunderstanding of staff rights. The study results also showed one third of nurses hint signals from others to leave their job ,this goes in line with Magee, 
Gordon,Caputi, Oades, Reis \&Robinson (2014) study in Australia that nurses have signals from other to quit their job.

According to physical intimidating bullying the study results indicated that more than one third of the studied nurses mentioned exposure to shout and impulsive anger. That result is consistent with El-Houfey, Abo El-Maged, Elserogy \& El Ansari (2015) study results which showed that more than half of the nursing team have been target of impulsive anger and shouting, also go in line with Esfahani \& Shahbazi (2014) in their study in West Azerbaijan, Iran which showed that more than one third of nurses occasionally target to verbal aggression and shouting. On the other extreme Yokoyama, Suzuki, Takai, Igarashi, Noguchi-Watanabe \& Yamamoto-Mitani (2016) results showed that minority were target to shout and anger. This result may be due to unacceptable level of violence from patients and their relatives, day-to-day work challenges with different patient and working with unsuitable physical environment.

The current study results also indicated that, about one third of the studied nurses exposed to intimidating behavior such as finger-pointing, invasion of personal space and shoving. This result is congruent with Roy\& Khan (2020) study which declared that nurses faced different intimidating behavior as finger-pointing. This is may be due to nursing profession is traditionally predominant female and nurses encountered frequently physical and nonphysical violence during work.

Concerning prevalence of workplace bullying among nurses the present study declared that more than half of studied nurses were victims to workplace bullying. This result goes along with Al-Ghabeesh \&Qattom (2019) who stated that most of nurses considered victims of bullying. In the same line study by Nwaneri, Onoka \&Onoka (2017) in Enugu, Nigeria found that majority of nurses were victims of workplace bullying according to definition of workplace bullying. Also agreed with Fontes, Santana, Pelloso \& Carvalho (2013) study in Brazil which revealed that nurses worked in public health care units were victims of workplace bullying .On other extreme Baburajan, Arasu \& Naveen (2019) who studied 300 staff nurses in Bangalore stated that only fifth of nurses has been bullied.

As regard perpetrators characteristics the present study results showed that, two third of nurses bullied by patients and patient relatives, as regard sex of perpetrator more than half of nurses bullied by both male \& female perpetrator. This result goes in line with Abbas, Fiala, 
AbdelRahman and Fahim (2010) who conducted study on nursing staff in four hospitals and twelve primary health care centers in Ismailia, Egypt and reported that most of nurses are bullied by patients and patients' relatives .Also, agree with study conducted in Egypt by Abdellah \& Salama (2017) which revealed that patients' relatives are the responsible for workplace violence. Furthermore, another study by El-Hneiti, etal.2019 found that more than one third of violent acts against nurses were perpetrated by patient's relative.

On the other extreme McMahon, MacCurtain, O’Sullivan, Murphy \&Turner (2013) study which revealed that the most frequent source of bullying is management predominately by supervisors or senior managers, also another study in Greece by Chatziioannidis, Bascialla, Chatzivalsama, Vouzas \&Mitsiakos(2018) found that majority of Perpetrators were female's supervisor and senior colleagues. This result may be due to high workload experienced by nurses that hinder nurses to provide required patient needs which rise physical workplace bullying from patient and their relatives toward nurses.

Regarding victimization profile the current study results showed that less than one quarter of studied nurses had one to six days off work due to being bullied, more than one third of nurses want to quit or transfer from present job due workplace bullying. This result goes in line with study in south-east of England by Quine (2001) which indicated that eight percent of bullied nurses had frequent sickness and absence, with more than three quarters thinking about quitting their job. Add to that Simons (2010) stated that victimized nurses are most likely to quit their jobs. This is may be due to bullying has a negative impact on nurses' physical and psychological health, resulting in frequent sickness and absence Kivimäki, Elovainio\&Vahtera (2000).

Current study results also showed that one eighth of nurses mentioned that their productivity sometimes lower than expected due to bullying. In the same line Berry, Gillespie, Gates, \& Schafer (2012) who studied registered nurse from Ohio, Kentucky, and Indiana indicated that nurse's productivity was negatively impacted by workplace bullying. This was contrasted to Elewa\& El Banan(2019) who stated that bullying had no effect on the productivity of one-half of the staff nurses . This may be due to nurses generally provide care in stressful areas with increased workloads and even in unfavorable stressful situations. 
The present result revealed that there was no statistical significant relation between workplace bullying and of socio-demographic characteristics of nurses. This result goes in line with Baburajan, Arasu \&Naveen (2019) who stated no significant relation between demographic characteristics and workplace bullying. Moreover, Alcantara, Claudio \& Gabriel (2017) revealed no significant association between personal demographic data and workplace bullying. In contrast to these results Alaslawi (2017) results declared weakened significant positive relation and Ariza- Montes, Muniz, Montero-Simo \& Araque-Padil (2013) ascertained relationship between workplace bullying and demographic data.

\section{CONCLUSION:}

It was concluded from this study, more than half of nurses were victims of workplace bullying, two third of nurses bullied by patients and patient relatives and more than half of nurses bullied by both male $\&$ female perpetrators. There was no statistical significant relation between workplace bullying and nurses socio-demographic data.

\section{RECOMMENDATIONS:}

- Counseling sessions about consequences of workplace bullying should be held on regular base in health care centers.

- Develop health education programs to nurse's about workplace bullying.

- Conduct workshops about workplace bullying prevention.

- Encourage nurses to report incidents of workplace bullying.

- Further research on workplace bullying with larger sample should be carried out.

\section{REFERENCES:}

Abbas, M. A., Fiala, L. A., Abdel Rahman, A. G., \& Fahim, A. E. (2010). Epidemiology of workplace violence against nursing staff in Ismailia Governorate, Egypt. The Journal of the Egyptian Public Health Association, 85(1-2), 29-43.

Abdellah,R. F., \& Salama, K. M. (2017). Prevalence and risk factors of workplace violence against health care workers in emergency department in Ismailia, Egypt. Pan African Journal, 26(21). https://doi.org/10.11604/pamj.2017.26.21.10837 
Alaslawi,H.A. (2017).WORKPLACE BULLYING IN KUWAIT.Published Doctoral Dissertation .Theses and Dissertations-Social Work. University of Kentucky 18.https://uknowledge.uky.edu/csw_etds/18

Alcantara, G. M., Claudio, E. G., \& Gabriel, A. G. (2017).Prognosis of Workplace Bullying in Selected Health Care Organizations in the Philippines, Open Journal of Social Science 5(9).154-174. http://doi.org/10.4236/jss.2017.59012

Al-Ghabeesh, S.H.,\& Qattom, H. (2019).Workplace bullying and its preventive measures and productivity among emergency department nurses. BMC health services research, 19(1), 445. https://doi.org/10.1186/s12913-019-4268-x

Al-Turki, N., Afify, A. A., \& AlAteeq, M. (2016). Violence against health workers in Family Medicine Centers. Journal of multidisciplinary healthcare,9(7), 257-266. https://doi.org/10.2147/JMDH.S105407)

Ariza-Montes,A., Muniz, N., Montero-Simo, M., \& Araque- Padil, R. (2013). Workplace Bullying among Healthcare Workers. International Journal of Environmental Research and Public Health, 10(8), 3121- 3139.

Baburajan,C., Arasu, S., \& Naveen, R. (2019). Prevalence of Bullying among Nurses in a Tertiary Hospital, Bangalore. International Journal of Occupational Safety and Health, 9(1), 8-12. https://doi.org/10.3126/ijosh.v9i1.25161

Berry, P.A., Gillespie, G.L., Gates, D. and Schafer, J.(2012). Novice Nurse Productivity Following Workplace Bullying. Journal of Nursing Scholarship 44(1).10.1111/j.1547-5069.2011.01436.x

Branch, S., Ramsay, Sh. \&Barker, M. (2013). Workplace Bullying, Mobbing and General Harassment: A Review. International Journal of Management Reviews (Review), 15 (3), 280-299. doi:10.1111/j.1468-2370.2012.00339.x.

Butler, E., Prentiss, A., \& Benamor, F.(2018).Exploring perceptions of workplace bullying in nursing. Nursing \& Health Sciences Research Journal, 1(1), 19-25.

Chatziioannidis I, Bascialla FG, Chatzivalsama P.,Vouzas,L.\& Mitsiakos,G.( 2018). Prevalence causes and mental health impact of workplace bullying in the Neonatal Intensive Care Unit environment. BMJ Open;8(2). doi: 10.1136/bmjopen-2017-018766. 
Ciby, M.\& Raya, R.P.(2015). Workplace bullying: a review of the defining features, measurement methods and prevalence across continents. IIM Kozhikode Society and Management Review, 4(1), pp. 38-47. https://doi.org/10.1177/2277975215587814

Duffy, M., \& Sperry, L. (2012). Mobbing: Causes, Consequences, and Solutions (1st edition.). USA: Oxford University Press, p70.

Einarsen, S., Hoel, H., \& Notelaers, G.(2009).Measuring exposure to bullying and harassment at work: Validity, factor structure and psychometric properties of the Negative Acts Questionnaire-Revised. Work \& Stress Journal, 23(1), 24-44.

Einarsen, S., Hoel, H., Zapf, D., \& Cooper, C. L. (2011). The concept of bullying and harassment at work: The European tradition. In S. Einarsen, H. Hoel, D. Zapf, \& C. L. Cooper (Eds.), Bullying and harassment in the workplace: Developments in theory, research, and practice (2nd Ed .,pp. 3-40). Boca Raton, FL: CRC Press.

Ekici,D., \& Beder, A. (2014). The effects of workplace bullying on physicians and nurses. Australian .Journal of Advanced Nursing, 31(4), 24- 33.

El Houfey A, Abo El Maged N, Elserogy Y and El Ansari W. (2015).Workplace Bulling Against Medical and Nursing Team Working At Emergency Departments in Assiut University Hospital. IOSR Journal of Nursing and Health Science; 4(2): 1-9.

Elewa ,A. \& El Banan,S.(2019) .Organizational Culture, Organizational Trust and Workplace Bullying Among Staff Nurses at Public and Private Hospitals .International Journal of Nursing Didactics. 9(4).10-20.

El-Hneiti, M., Shaheen, A. M., Bani Salameh, A., Al-Dweeri, R. M., Al-Hussami, M., Alfaouri, F. T., \& Ahmad, M. (2019). An explorative study of workplace violence against nurses who care for older people. Nursing open,7(1), 285-293. https://doi.org/10.1002/nop2.389

Esfahani, A. N., \& Shahbazi, G. (2014). Workplace bullying in nursing: The case of Azerbaijan province, Iran. Iranian journal of nursing and midwifery research, 19(4), 409415.

Fontes, K. B., Santana, R., G., Pelloso, S., M., \& Carvalho, M.,D. (2013). Factors associated with bullying at nurses' workplaces. Revista Latino-Americana de Enfermagem, 21(3), 758-764. 
Ganz, F.D., Levy „H., Khalaila „R., Arad, D., Bennaroch, K., Kolpak, O., et al. (2015), Bullying and its prevention among intensive care nurses. Journal of Nursing Scholarship 47(6) 505-511. doi: https://doi.org/10.1111/jnu.12167

Hansen, A. M., Hogh, A., Garde, A. H., \& Persson, R. (2014). Workplace bullying and sleep difficulties: A 2-year follow-up study. International Archives of Occupational and Environmental health, 87(3), 285-294.doi: 10.1007/s00420-013-0860-2.

Howell, C. J. (2016). Influencing Factors Related to Workplace Bullying Among Nurses: A Systematic Review.Published master's thesis, University of Alberta, Edmonton,Canada.Availablehttps://scholar.google.com.eg/scholar?hl=en\&as_sdt= $0 \% 2 \mathrm{C} 5 \& \mathrm{q}=$ Influencing+Factors+Related+to+Workplace+Bullying+among+Nurses $\% 3 \mathrm{~A}+$ $\mathrm{A}+$ Systematic+Review\&btnG=https://uknowledge.uky.edu/cgi/viewcontent.cgi? $\operatorname{article}=10$ $18 \&$ context=csw_etds.

Johnston,M., Phanhtharath, P., \& Jackson, B.S. (2013). The bullying in nursing. Critical Care Nursing Quarterly, 32(4), 287-295.

Jones, A. (2017). Experience of Protagonists in Workplace Bullying: An Integrated Literature Review Publication History: Keywords. International Journal of Nursing and Clinical Practice, 4 (1), 246.10.15344/2394-4978/2017/246.

Karatza, C., Zyga, S., Tziaferi, S., \& Prezerakos, P. (2017). Workplace Bullying Among the Nursing Staff of Greek Public Hospitals. Workplace Health \& Safety, 65(2), 57-64. https://doi.org/10.1177/2165079916657106

Kivimäki M, Elovainio M, Vahtera J. (2000). Workplace bullying and sickness absence in hospital staff. Occup Environ Med;57(10):656-60.

Lawn, J.E., Rohde, J., Rifkin, S., Were, M., Paul, V.K., \& Chopra M. (2008) AlmaAta 30 years on: revolutionary, relevant, and time to revitalise. The Lancet, 372(9642):917-927.

Lee, Y.J., Bernstein, K., Lee, M., \& Nokes, K. (2014). Bullying in the nursing workplace: Applying evidence using a conceptual framework. Nurs Econ; 32(5):255-267.

Magee,C., Gordon,R., Caputi,P., Oades,L., Reis,S., \&Robinson,R.(2014) Final Report Workplace Bullying in Australia.Centre for Health Initiatives at the University of Wollongong. 
McMahon, J., MacCurtain, S., O’Sullivan, M., Murphy, C, \& Turner, T. (2013). A Report on the Extent of Bullying and Negative Workplace Behaviours Affecting Irish Nurses. Dublin: Irish Nurses and Midwifery Organisation (INMO).

Nielsen, M. B., Matthiesen, S. B., \& Einarsen, S. (2010). The impact of methodological moderators on prevalence rates of workplace bullying. A meta-analysis. Journal of Occupational and Organizational Psychology, 83(4), 955-979. https://doi.org/10.1348/096317909X481256

Nwaneri, A,C.Onoka,A.C., \&Onoka ,C.A.(2017).Workplace bullying among nurses working in tertiary hospitals in Enugu, southeast Nigeria: Implications for health workers and job performance. Journal of Nursing Education and Practice7 (2), 69-78. doi:10.11604/pamj.2017.26.21.10837.

Quine, L. (2001). Workplace bullying in nurses. Journal of Health Psychology, 6(1), 73-84.

Rai, A. \& Agarwal, U. (2017) .Linking workplace bullying and work engagement: the mediating role of psychological contract violation. South Asian Journal of Human Resources Management 4(1), 42-47. 10.1177/2322093717704732

Roy,L. \& Khan.,M.(2020) Workplace bullying among nurses in Bangladeshi government hospitals.Asian Journal of Medical and Biological Research. 6 (1), 67-72; doi: 10.3329/ajmbr.v6i1.46480.

Salin, D. (2015). Risk factors of workplace bullying for men and women: The role of the psychosocial and physical work environment. Scand J Psychol. 56(1), 6977.https://doi.org/10.1111/sjop.12169

Sauer,P. A., \& McCoy, T. P. (2017). Nurse bullying: Impact on nurses' health. Western Journal of Nursing Research, 39(12), 1533 1546. https://doi.org/10.1177/0193945916681278

Simons, S.R. (2010) .Workplace bullying experienced by nurses newly licensed in Massachusetts and the relationship to intention to leave the organization. Ph.D. dissertation, University of Massachusetts Boston. Available at: http://proquest.com (Publication No. AAT3221377). 
Spector, P.E., Zhou ,Z.E., \& Che, X.X. (2013).Nurse exposure to physical and nonphysical violence, bullying, and sexual harassment: A quantitative review. International Journal of Nursing Studies. 51(1):72-84. DOI: 10.1016/j.ijnurstu.2013.01.010 [PubMed: 23433725]

Tehrani, N. (2013).workplace bullying symptoms and solutions (1st edition.). New York, Routledge Company of the Taylor \& Francis Group, p4.

World Health Organization.(2012).Workplace violence. Retrieved from https://www.who.int/violence_injury_prevention/injury/work9/en/ (accessed in May 2017).

Wright, W., \& Khatri, N. (2015). Bullying among nursing staff: Relationship with psychological/behavioral responses of nurses and medical errors. Health Care Management Review, 40(2),139-147, doi: 10.1097/HMR.0000000000000015

Yokoyama, M., Suzuki, M., Takai, Y., Igarashi, A., Noguchi-Watanabe, M., \& Yamamoto-Mitani, N. (2016). Workplace bullying among nurses and their related factors in Japan: A cross sectional survey. Journal of Clinical Nursing, 25(17), 24782488. https://doi.org/10.1111/jocn.13270

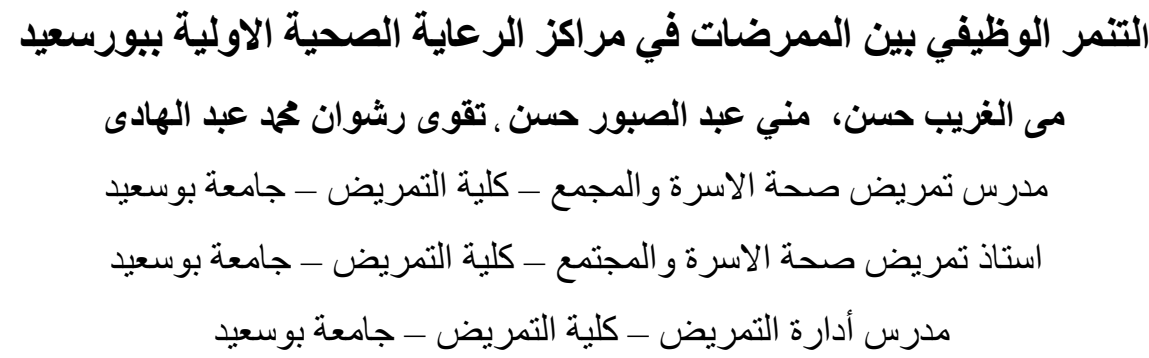

\section{الخلاصة}

التتمر الوظيفي مشكلة شائعة تهدد مهنة التمريض وصحة الممرضات وتؤثر علي قدرتهن على العمل بأمان ولذلك قد هدفت الدراسة الي تقييم التنمر الوظيفي بين الممرضات بمر اكز الرعاية الصحية الأولية بمحافظة بورسعيد. أجريت هذه الدر اسة في سبع مر اكز للرعاية الصحية الأولية والتي مثلت احياء محافظة بورسعيد.وقد اشتملت العينة علي جميع الممرضات (61) العاملات في العيادات المختلفة في مراكز الرعاية الصحية الأولية التي تم اختبارهم بصورة عشو ائية. تم جمع البيانات من خلال استمارة استبيان الأفعال السلبية المنقح ,وقد اوضحت النتائج ان غالبية الممرضات (86.9\%) حصلو ا على تعليم ثانوي تمريض و 82\% من الممرضات متزوجات.كما اوضحت النتائج ان أكثر من نصف الممرضات الخاضعين للار اسة (54.1٪) هم ضحايا للتنمر الوظيفي في مراكز الرعاية الصحية الأولية ، وقد تعرض ثلثي الممرضات (60.6٪) للتنمر من قبل المرضى و أقارب المرضى بينما اوضحت النتائج ان أكثر من الثلث (33.3٪) قد تعرضوا للتنمر الوظيفي من قبل المشرفين و المدراء.علاوة علي ذلك لم تكن هناك علاقة ذات دلالة إحصائية بين التنمر الوظيفي والبيانات الاجتماعية والديموغر افية للمرضات.لذا اوصت الدراسة بضرورة إجراء جلسات تتتقفية عن عو اقب التنمر الوظيفي علي الممرضات بصفة دورية في مر اكز الرعاية الصحية الاولية. الكلمات المرشدة: التنمر الوظيفى ، الممرضات ، مر اكز الرعاية الصحية الأولية 